\title{
An Individualised Learning and Exercise Program Based on the Bobath Concept to Facilitate Goal Achievement in People with Chronic Stroke
}

\author{
Eckhardt Gabriele $^{1}$, Brock Kim ${ }^{2}$, Haase Gerline ${ }^{1}$, Ishida Toshie ${ }^{3}$, Hummelsheim Horst ${ }^{4}$ \\ ${ }^{1}$ Center of Physiotherapy and Rehabilitation, Haan, Germany \\ ${ }^{2}$ St Vincent's Hospital, Melbourne, Australia \\ ${ }^{3}$ Juntendo University Nerima Hospital, Tokyo, Japan \\ ${ }^{4}$ Neurological Rehabilitation Center-Leipzig, Leipzig-Bennewitz, Germany
}

Email address:

g.eckhardt@burgerland.de (E. Gabriele)

${ }^{*}$ Corresponding author

\section{To cite this article:}

Eckhardt Gabriele, Brock Kim, Haase Gerline, Ishida Toshie, Hummelsheim Horst. An Individualised Learning and Exercise Program Based on the Bobath Concept to Facilitate Goal Achievement in People with Chronic Stroke. American Journal of Health Research. Vol. 9, No. 1, 2021, pp. 26-33. doi: 10.11648/j.ajhr.20210901.14

Received: February 15, 2021; Accepted: February 24, 2021; Published: March 9, 2021

\begin{abstract}
Background: To facilitate improvement in participation, therapy needs to be transferred into everyday life of people with chronic stroke. Individualised, home based, self-training exercise programs should be oriented towards the potential of the person, be specific to their ability and impairment levels, avoid compensation strategies as far as possible and operate at the upper limit of the individual's ability level. Objectives: To investigate the effectiveness of an individualised learning and exercise program based on the Bobath concept for facilitating personal goal achievement in people with chronic stroke. Method: Repeated measures design. Fifty-five people with chronic stroke were recruited from participants in Advanced Bobath training courses. During the five day course, participants learned individualised self-training programs, which they then carried out at home for three months, adapting their program according to their performance level. The primary outcome measure was the Canadian Occupational Performance Measure (COPM) - Performance domain. Secondary measures included the COPM satisfaction domain, World Health Organisation Disability Assessment Score-2 (WHO-DAS2), and Goal Attainment Score (GAS). Measurements were taken three months and immediately prior to the Bobath course and three months after the course. Results: COPM-Perfomance, COPM-Satisfaction and the WHODAS-2 showed a significant difference over the time points, with followup scores significantly greater than both baseline and preintervention $(p<.001)$. Sixty eight percent of participants achieved their personal goals (GAS). Conclusion: The study indicates that an individualised self-training approach based on Bobath principles may enable the transfer of acquired proficiency into the personal goals of people with chronic stroke.
\end{abstract}

Keywords: Bobath, Individual Self Training, Exercise Program, Stroke

\section{Introduction}

There is growing interest in rehabilitation interventions extending beyond the subacute phase for stroke survivors [1,2] underpinned by studies showing poor outcomes for quality of life and participation $[3,4]$. There is evidence that stroke survivors can benefit from physiotherapy in the chronic phase, however, the costs of providing this care are considerable [5].
The use of individually tailored self training in a home based exercise program has yielded positive results for increasing physical activity [6], walking capacity $[7,8]$ and upper limb function [9]. There is less evidence for improving participation, including achievement of personal goals. Goal directed, individualised self training programs may be a useful approach for enhancing achievement of personal goals in stroke survivors.

The Bobath concept is a well established approach to 
neurorehabilitation. The clinical reasoning of the Bobath therapist involves a complex assessment focusing on the analysis of movement behaviour in task performance [10]. The therapist assesses the quality and efficiency of movement, the individual impact of the neurological condition on the person and identifies potential for more efficient movement to enable achievement of meaningful goals [10]. In Bobath practice, interventions are necessarily individualised; this applies equally to the therapist/ patient interaction and development of self training programs. In this context, the question arises as to whether an individualized self training program, developed in accordance with Bobath principles, will bring about improvements in participation in stroke survivors.

The primary hypothesis for this pilot study is that participants with chronic stroke who receive a combined intervention of individualised Bobath training and a goal directed, self training program will improve more in their ability to perform their own personal goals, as measured by the Canadian Occupational Performance Measure (COPM) Performance scale [11], compared to a baseline period.

Secondary hypotheses are that greater improvements will also be observed compared to a baseline period for the following domains

a) Satisfaction with ability to perform personal goals [11] (COPM Satisfaction scale)

b) Self reported disability (WHO Disability Assessment Scale, version 2 [12] (WHO-DAS 2) and

c) Physical function, as measured by the Rivermead Mobility Index [13] (RMI)

\section{Method}

Design: Repeated measures study. This project received ethical approval from Human Research Ethics Committees in Germany (Ethics Committee at Physio-Akademie of the German Physiotherapy Association (ZVK e.V.)) and Japan (Ethical Review Board of Juntendo University Nerima Hospital), conforming to the Declaration of Helsinki.

Written informed consent was gained from all participants.

Setting: Three ambulatory rehabilitation services in Germany and one outpatient service in an acute neurological hospital in Japan.

Participants: People with chronic stroke who had agreed to participate in a five day Advanced Bobath training course for physiotherapists and occupational therapists were invited to participate in the study. In Advanced Bobath training courses certified by the International Bobath Instructor Training Association (IBITA), people with neurological diagnoses attend for daily 90 minute assessment and treatment sessions conducted by course participants (qualified physiotherapists and occupational therapists), under the supervision of course tutors (certified by IBITA). The course venue is responsible for inviting current and previous patients to participate in the Bobath course. The selection criteria for patients attending a Bobath course with the self training theme included chronic stroke, significant sensori-motor dysfunction, limitations at an activity and participation level and able and willing to participate in therapy for daily 90 minutes treatment sessions.

The inclusion criteria for this study were

1. Moderate disability following stroke

2. A minimum of 12 months post stroke

3. Cortical stroke

4. Friends or relatives living in the same household

Exclusion criteria were

1. Pronounced receptive and/or expressive speech deficits

2. Pronounced neglect (Catherine Bergego Scale14 > 20),

3. Unable to follow a two-step command,

4. Full dependency in all ADLs

Intervention

The intervention consisted of two parts. The first part involved the development of a personalised self training program together with the therapist during the five day Bobath course. In the second part, the participants carried out their self training program with or without the help of carers at home over a three month period (see Figure 1).

In the first part, participants attended the Advanced Bobath Course, receiving 90 minutes of Bobath therapy on five consecutive days. The focus of these therapy sessions was the development and learning of a self training program. The self training program was designed to meet the following criteria:

a) developed in response to the person's own goals:

b) adapted to the individual's ability and neurological impairment

c) developing the potential of the person for further improvement

d) minimising the practice of compensatory strategies [15]

e) using their usual personal environments and equipment already available to them

On commencement in the Advanced Bobath Course, each participant was assessed with regard to their personal situation, movement dysfunction and impairments. Together with the participant, personal goals were identified with potential for achievement through the methodical and systematic application of an individualised exercise program. Personal goals were structured into the SMART goal format [16]. The therapist then divided the desired goal into activities, movement sequences, and movement components. Movement analysis provided information about the sequences and components of movement that the person could not complete or where movements were not efficient or economical. For the desired action, the patterns of activation of both target muscles and compensatory muscles were evaluated. The assessment also focused on the impairments underlying the movement problems; the relative proportions and severity of motor, sensory, biomechanical, perceptual and/or cognitive and emotional disorders [17]. The therapist formulated working hypotheses as to the underlying causes of the movement problem [10], these were then tested in the treatment sessions during the five day course and adapted and changed as necessary. Once the therapists had confirmed a treatment approach that yielded improved performance within session, exercises were developed for the person to practice at home. 
The exercises focused on specific movements, aimed at improving control of movement and avoiding the use of compensatory strategies. The exercises also included components related to specific impairments, for example, inclusion of exercises involving turning to improve orientation in space in patients with perceptual issues, or exercises to mobilise the hip in patients with motor and biomechanical impairments. Programs usually included both part task and whole task exercises and exercises outside the task where specific components were addressed in different postures or alignments. Other postures were utilised in order to make it easier to achieve the movement component; for example, working on core stability aspects in supine or sidelying.

For the exercise program, a folder with photos was used; each folder was created in the Advanced Course together with the patient participants, inclusive of written instructions and comments (see Online Supplement for examples of exercise programs). Information was provided on how to perform the exercise and recommendations for the number of repetitions. In addition, three variations of each exercise ( $a, b$ and $c)$ were developed so the patient could adapt the exercise to increase or decrease the difficulty level, allowing shaping of the exercise to their ability level on a specific day. In order to move on to a more difficult version of the exercise, the patient had to reach specified levels of ability. For some patients, a relative or friend provided cueing, supervision or light assistance to enable practice of difficult components.

In the second part, the participant was asked to carry out their individualised program for three months, including adapting their exercises according to their own assessment of their performance. A calendar was used over the duration of the second part to document the level of difficulty performed for each exercise ( $a, b$ or $c$ ), enabling the participant to visualize their achieved level of performance.

In five Advanced Courses, 59 therapists (36 PT, 23 OT) were trained to create the individualized self training programs. Four IBITA recognized advanced course instructors were involved in providing the training.

\subsection{Measures}

The primary dependent variable was the Canadian Occupational Measurement (COPM) Performance domain [11]. The COPM is a person centered measure where a structured interview is conducted to identify daily activities that the person wants to do, needs to do or is expected to do but is unable to accomplish. The COPM Performance domain measures the person's self perceived level of ability to perform the identified task. In the Satisfaction domain, the person rates their satisfaction with their performance level. For this study, COPM Performance was chosen as the primary outcome as it measures the person's ability to perform their own goals, ensuring that outcomes achieved are significant and relevant to the person. Reliability and validity of COPM has been demonstrated [18]. The COPM has been widely utilized to measure the effectiveness of interventions following stroke [19-24].

Secondary variables include the World Health Organization Disability Assessment Score 2.0 [12] (WHO DAS-2), and the Rivermead Mobility Index (RMI). The WHO DAS-2 has undergone extensive psychometric testing [25]. Reliability [13] and aspects of validity and responsiveness $[26,27]$ have been established for the RMI.

Measurements were taken at baseline (three months before commencing the Advanced course), immediately prior to the Advanced course and three months after completing the course (see Figure 1). An independent rater, physiotherapist, occupational therapist or neuropsychologist from each center conducted the assessments; raters were not involved in the study design, selection of participants, or delivery of the intervention. The raters were trained in the assessment procedures. The baseline and pre-course assessments were completed in person and the follow up assessments were completed in person or by telephone.

In addition to these measures, Goal Attainment Scaling [28] (GAS) was utilized during the Advanced Courses to help participants and therapists clarify goals and the level of achievement the stroke survivors were aiming for. GAS ratings achieved were scored during the follow up assessment by the independent raters.

\subsection{Statistical Analysis}

As an initial step, the variables of interest were investigated for normality of distribution by visual inspection and analysis of skewness and kurtosis. Analysis of variance for repeated measures (ANOVA) was utilized to test for significant differences between measurement times. Where significant differences were identified, post hoc analysis with the least significant difference test was used to identify the time points with significant differences. Post hoc power analysis was utilized to determine the sample size required for a definitive trial.

\section{Results}

Fifty five people with stroke participated in the study. Demographic and medical variables of participants are presented in Table 1. Fifty four of the 55 participants participated in assessments at the three time points (Figure 1). Each participant with stroke had a self training program folder, containing an average of 8.98 individually devised exercises (SD 2.56, range $4-17$ ) in relation to their personal goals. In all, 503 individual exercises were developed and utilized. On average, participants carried out all or part of their program on 73.04 of the 92 days (SD 19.85 Range 2-92). Table 2 shows baseline, preintervention and followup data for all outcome variables. 
Table 1. Demographic and medical variables at baseline.

\begin{tabular}{ll}
\hline Gender & Men 25 \\
Age & Mean 56.87 (SD 13.29; range 26 -82) \\
Side of hemiparesis & Right 24, Left 31 \\
Type of stroke & Infarct Haemorrhage \\
Time since injury & 7.12 years (SD 6.67; range 1-27 years) \\
Berg Balance Score Maximum score 56 & Mean 45.1 (SD 11.7; range 9 -56) \\
MESUPES* Arm Maximum score 40 & Mean 19 (SD10.3; range 0 -39) \\
MESUPES Hand Maximum score 12 & Mean 3.3 (SD4.5; range 0-12) \\
MESUPES Orientation Maximum score 6 & Orientation 0.8 (SD1.6; range 0-6) \\
\hline
\end{tabular}

MESUPES: Motor Evaluation Scale for Upper Extremity in Stroke.

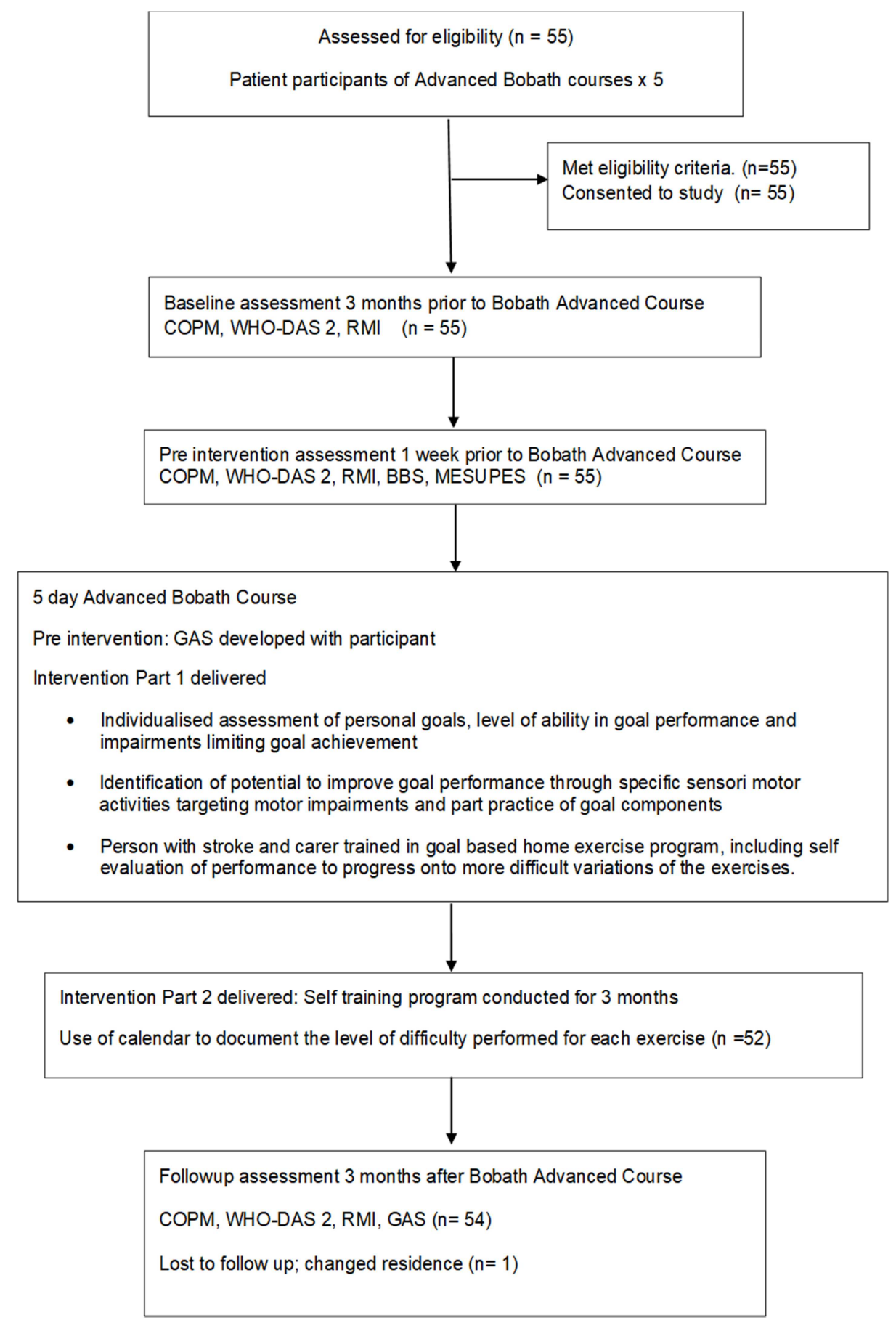

Figure 1. Flow chart participants. 
Table 2. Primary and secondary variable scores at three measurement points.

\begin{tabular}{llll}
\hline & Baseline Mean (SD) & Pre-Intervention Mean (SD) & Follow up Mean (SD) \\
\hline COPM - Performance & $3.3(1.52)$ & $3.5(1.54)$ & $4.61(1.6)$ \\
COPM - Satisfaction & $2.56(1.47)$ & $2.79(1.68)$ & $4.3(1.86)$ \\
WHO DAS-2 & $27.75(16.7)$ & $27.45(15.98)$ & $23.08(15.14)$ \\
Rivermead Mobility Index & $11.67(2.89)$ & $11.91(2.55)$ & $12.37(2.62)$ \\
\hline
\end{tabular}

Prior to conducting the comparative analysis, visual inspection of boxplots at the three measurement time points demonstrated normal distribution patterns for all variables (Figure 2). For the primary variable of COPM Performance Score, repeated measures ANOVA showed that the mean values for the three measurement points were significantly different $F(2.11)=29.69, p<0.001$. Post-hoc analyzes using the least significant difference test demonstrated that the COPM Performance score at the follow-up measurement point was significantly higher than at the baseline and pre-intervention measurement points, $\mathrm{p}<0.001$, (Figure 2a). No significant difference was found between baseline and pre-intervention $(\mathrm{p}=.16)$.

\subsection{Secondary Variables}

For COPM satisfaction, the ANOVA showed that the mean

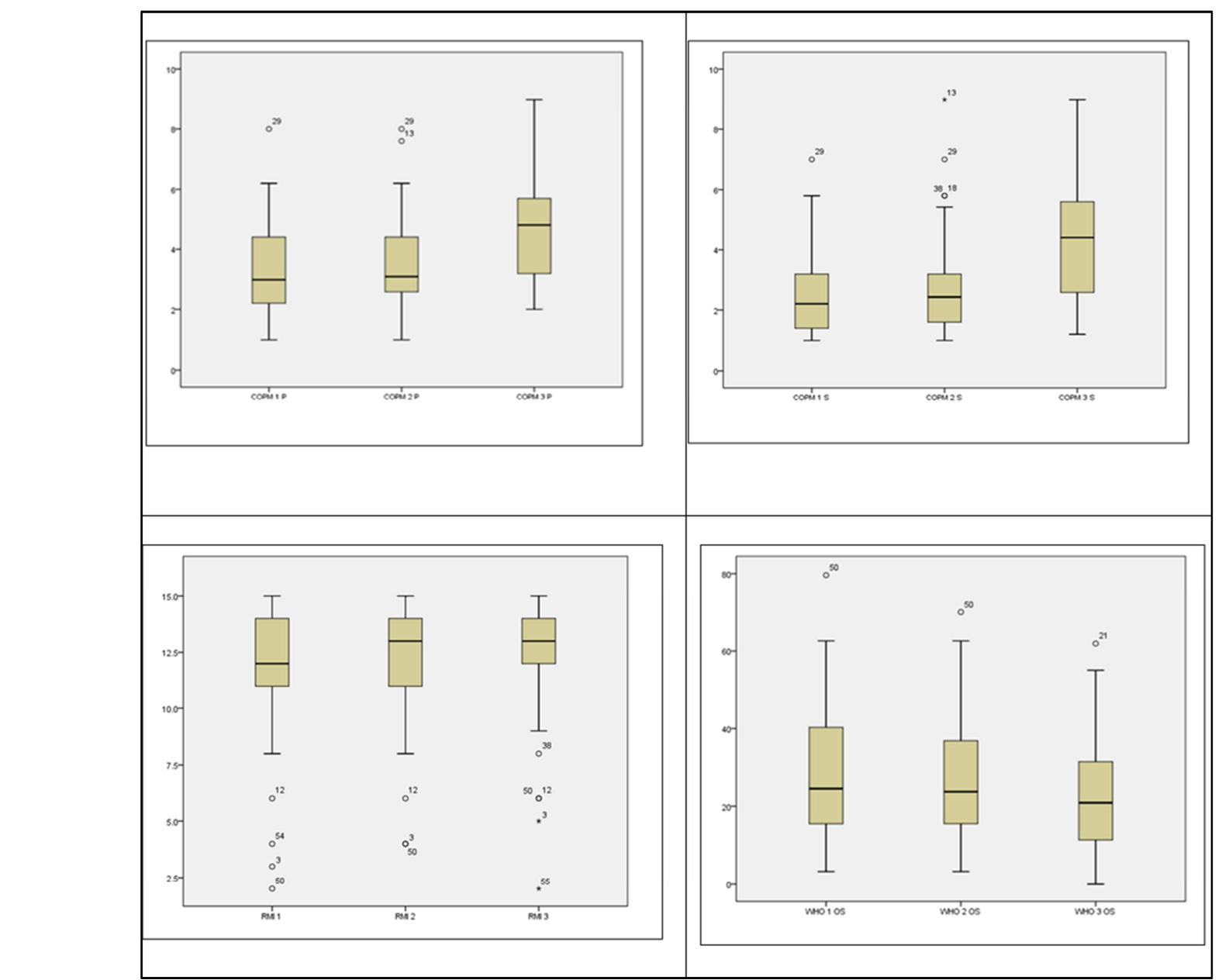

Figure 2. Boxplots for primary and secondary measurements at three time points; 3 months prior, one week prior and three months post the 5 day Bobath course a) Candian Occupational Performance Measure-Performance, b) Candian Occupational Performance Measure-Satisfaction, c) Rivermead Motor Index, d)

WHO Disability Assessment Scale-2. values for the three measurement points were significantly different $F(2,106)=45.018, p<0.001$ (Figure 2b). Post-hoc analyzes demonstrated that the COPM satisfaction score at the follow-up measuring point was significantly higher than at the baseline and pre-intervention measuring points, $p<0.001$. No significant difference was found between baseline and pre-intervention $(\mathrm{p}=.14)$.

For the WHO DAS-2, the ANOVA showed that the mean values for the three measurement points were significantly different $F(2,106)=12.44, \mathrm{p}<0.001$ (Figure 2c). Post-hoc analyzes demonstrated that the WHO DAS-2 score at the follow-up measurement point was significantly lower than the baseline and pre-intervention measurement points, $\mathrm{p}=0.001$. No significant difference was found between baseline and pre-intervention $(\mathrm{p}=.5)$. 
For the Rivermead Mobility Index, the ANOVA showed that the mean values for the three measurement points were significantly different $F(1.36 .73)=8000, p<0.003$ (Figure 2d). Post-hoc analyzes demonstrated that each comparison between time points was significant (baseline to pre-intervention $\mathrm{p}=.027$; baseline to follow up $\mathrm{p}=.002$; and pre-intervention to follow up $\mathrm{p}=.019$ ).

Fifty three participants rated their goal achievement level at the follow up assessment according to the Goal Attainment Score (Table 3$)$. Sixty eight percent of all participants $(\mathrm{N}=36)$ achieved their goal $(0)$ or were better than expected $(+1,+2)$.

Table 3. Goal Attainment Score $(N=53)$.

\begin{tabular}{lllll}
\hline-2 & -1 & 0 & +1 & +2 \\
9 & 8 & 19 & 10 & 7 \\
\hline
\end{tabular}

\subsection{Sample Size Calculation}

Sample size calculations for a definitive trial utilized differences in change scores over time for the COPM Performance domain. With alpha set at 0.05 , power at 0.8 and a two-tailed test, 33 participants per group are required for a definitive trial.

\section{Discussion}

The results of the study indicate that people with chronic stroke may improve their ability to perform activities of personal importance, as measured by the COPM performance, following an individualized self training program based on the Bobath concept, conducted over a three month period. The self training programs were developed during an intensive five day therapy program and were individually tailored, based on participants' personal goals, individual needs, abilities and neurological impairments. The intensive therapy period focused on the participant becoming more aware of their own performance and therefore able to monitor the success or otherwise of their practice during the self training period. Two thirds of the participants achieved their own personal GAS goals.

The secondary measures of COPM satisfaction and WHO DAS-2 also showed improvement following the intervention. The lower scores observed in the WHO DAS-2 indicate that self perceived disability had reduced. For the Rivermead Mobility Index, improvement was demonstrated between each of the assessments. This was an unexpected finding of the study, however, the differences were small (average change less than one point on a 15 point scale).

Previous studies investigating self training have focused on measures of function such as walking capacity [8] or upper limb function [9]. This study indicates that an individualised self training program may also assist with performance in relevant activities when personal goals are targeted, potentially reducing participation restriction.

The research project set a time limit of 92 days for the self training program. Patients were encouraged to carry out their program as often as possible. The optimal dosage of training days or training times in self training in neurorehabilitation is not known [29]. Some guidelines suggest 150-minutes of exercise a week $[30,31]$. In the present research, the participants practiced on average 73 days, showing a high motivation to use their exercise program. However, the amount of time spent on each exercise occasion was not recorded. In further research, this question should be considered. It is possible that a goal directed, individualized self training program makes the relevance of the exercises more understandable, increases the willingness to practice, and thus encourages the number of repetitions required for the learning process.

\section{Limitations of the Study}

The study has a number of limitations in the study design. Participants acted as their own controls in this repeated measure design; use of randomization would assist in determining the efficacy of this approach. Also, the participants were recruited from patients selected to participate in a training course for experienced therapists. It is not known how representative this group is of people with chronic stroke. The distribution of Berg Balance Scores indicate that the group did demonstrate a spread of ability associated with moderate stroke. Future studies should consider strategies to ensure a representative sample.

All participants were able to take part in the structured interview for the COPM, identify goals in everyday life of importance to them and rate both performance ability and satisfaction. In comparison, the use of GAS was more problematic in the study, with extensive training required to equip therapists with the ability to set person centered, relevant goals on the five point scale, adhering to SMART guidelines [17]. Establishing the five levels of goal achievement also required therapists to make judgements about potential to improve, which may be more or less accurate, to support the person with stroke to identify different levels of achievement. The COPM may have advantages over GAS in that less is required from both therapist and participant in determining potential to improve; and the person themselves determines the achievement level.

It should be noted that the average change scores between the pre-intervention and follow up measures was lower than the minimum detectable change for COPM Performance of 1.7 points 18. Self training exercises are a component of behavioural self management [2]. The concurrent use of other interventions recommended for behavioural self management, such as identification of barriers and problem solving, with ongoing support, may yield greater change in COPM scores.

In this study, 503 exercises related to personal goals and individual ability and impairment were developed and documented. These exercises form a rich resource for content analysis, to unpack the therapy component provided, in order to elaborate the principles utilized to construct and individualize interventions. The authors are currently conducting these qualitative analyses to inform clinicians 
interested in exploring this approach and to assist replication of the study.

\section{Conclusion}

The results of this study indicate that an individualised self training approach based on the principles of the Bobath concept may enable the transfer of acquired proficiency into the personal goals of people with chronic stroke. The results of this pilot study can be utilized to inform further clinical trials investigating the effectiveness of this approach. Consideration should be given to complementary interventions to further enhance outcomes.

\section{Conflict of Interest Statement}

The authors declare that they have no competing interests.

\section{Acknowledgements}

The authors would like to thank all the course participants and patients who volunteered to participate in this study. Thank you Valeska Benz, Angelika Eigen, Friederike Goslar, Heidi Lessig, Oki Mayuka, Linzi Smith-Meadows, and Hiromi Sato for your support. Thanks to the members of the Science Advisory Board of the "Vereinigung der Bobath Therapeuten Deutschland" for your encouragement and specially Karoline Munsch for your professional proofreading and suggestions.

Partial funding for this study was provided by an IBITA Research Grant.

\section{References}

[1] Graven, C., Brock, K., Hill, K., \& Joubert, L. (2011). Are rehabilitation and/or care co-cordinatiin intervention delivered in the community effective in reducing depression, facilitation participation and improving quality of life after stroke? Disabil Rehabil, S. 33 (17-18): 1501-20.

[2] Dobkin, B.-H. (2016). Behavioral self-management strategies for practice and exercise should be included in neurologic rehabilitation trials and care. Curr Opin Neurol, S. 29: 693699.

[3] Mayo, N., Wood-Dauphinee, S., Côté, R., Durcan, L., \& Carlton, J. (Aug 2002). Activity, participation, and quality of life 6 months poststroke. Arch Phys Med Rehabil, S. 83 (8): $1035-42$

[4] van Mierlo ML \& van Hoogstraten.-M. (2016). Quaility of Life during the first two years post stroke: The restore 4 Stroke Cohort Study. Cerebrovasc Dis., S. 41 (1-2): 19-26. doi: $10.1159 / 000441197$.

[5] Ferrarello F, Baccini M, Rinaldi LA, et al. Efficacy of physiotherapy interventions late after stroke: A meta-analysis. J Neurol Neurosurg Psychiatry. 2011; 82 (2): 136-143. doi: 10.1136/jnnp.2009.196428.

[6] Morris, J., MacGillivray. S, \& Mcfarlane. S. (2014).
Interventions to promote long term particpation in physical activity after stroke: a systematic review of the literature. Archieves of Physical Medicine and Rehabilitation, S. 95: 956-67.

[7] Brazzelli, M., Saunders, D., Greig, C., \& Mead, G. (Nov 2011). Physical fitness training for stroke patients. Cochrane Database Syst Rev., S. 9; (11): CD003316. doi: 10.1002/14651858.CD003316.pub4.

[8] Treger I, Landesman C, Tabacaru E, Kalichman L. Influence of home-based exercises on walking ability and function of post-stroke individuals. Int J Ther Rehabil. 2014; 21 (9): 441-446. doi: 10.12968/ijtr.2014.21.9.441.

[9] Rand, D., Weingarden, H., Weiss, R., et al (2017). Self-training to improve UE function at the chronic stage post-stroke: a pilot randomized controlled trial. Disabil Rehabil., $39 \quad$ (15): $\quad 1541-1548 . \quad$ doi: 10.1080/09638288.2016.1239766. Epub 2016 Oct 28 .

[10] Michielsen, M., Vaughan-Graham, J., Holland, A., Magri, A., \& Suzuki, M. (2017). The Bobath concept - a model to illustrate clinical practice. Disabil and Rehab, https://doi.org.10.1080/09638288.2017.1417496.

[11] Law, M. B. (2005). Canadian Occupational Performance Measure (4th ed.).. Ottawa, ON: CAOT Publications ACE.

[12] Üstün, T., Chatterji, S., Kostanjsek, N., Rehm, J., \& al., (2010). Developing the World Health Organisation Developing Schedule 2.0. Bull World Health Organ (88: 815-823).

[13] Collen, F., Wade, D., Robb, G., \& \& Bradshaw, C. (1991). The Rivermead Mobility Index: A further development of the Rivermead Motor Assessment. Intern Disabil, Vol 13, 50-54.

[14] Azouvi, P. (2017). The ecological assessment of unilateral neglect. Annals of Physical and Rehabilitation Medicine, 60 (3), 186-190. https://doi.org/10.1016/j.rehab.2015.12.005.

[15] Vaughan-Graham, J., \& Cott, C. (2016). Defining a Bobath clinical framework-a modified e-Delphi study. Physiother Theory Pract, S. 32 (8): 612-627.

[16] Bovend' Eerdt, TJ; Botell, R E, Wade, DT (2009). Writing SMART rehabilitation goals and achieving goal attainment scaling: a practical guide. Clinical Rehabilitation (23: 352-61).

[17] Eckhardt G, Brock K, Haase G, Puschnerus C, Hengelmolen-greb A, Böhm C. Bobath Concept Structural Framework (BCSF): Positioning Partial Aspects Within a Holistic Therapeutic Concept. 2018; 6 (4): 79-85. doi: 10.11648/j.ajhr.20180604.11.

[18] Cup, EH; Scholte op Reimer, WJ.; Thijssen, M., \& van Kuyk-Minis, M. (2003). Reliability and validity of the Canadian Occupational Performance Measure in stroke patients. Clin Rehabil., 17 (4): 402-9.

[19] Lund, A. M. (2012). Development of a person-centred lifestyle intervention for older adults following a stroke or transient ischaemic attack.. Scandinavian Journal of Occupational Therapy, 19 (2), 140-149.

[20] McCall, M. M. (2011). Modified constraint-induced movement therapy for elderly clients with subacute stroke. The American Journal of Occupational Therapy, 65 (4), 409-418. 
[21] Mew, M. (2010). Normal movement and functional approaches to rehabilitate lower limb dressing following stroke: A pilot randomised controlled trial.. British Journal of Occupational Therapy, 73 (2), 64-70.

[22] Nilsen, D. M. (2012). Effect of imagery perspective on occupational performance after stroke: A randomized controlled trial.. The American Journal of Occupational Therapy, 66 (3), 320-329. doi: 10.5014/ajot.2012.003.

[23] Roberts, P. S. (2005). Client-centered occupational therapy using constraint-induced therapy. Journal of Stroke and Cerebrovascular Diseases, 14 (3), 115-121.

[24] Shaw, L. R. (2010). BoTULS: A multicentre randomised controlled trial to evaluate the clinical effectiveness and cost-effectiveness of treating upper limb spasticity due to stroke with botulinum toxin type A. Health Technology Assessment, 14 (26), 1.

[25] WHO DAS manual. (May 2018) http://apps.who.int/iris/bitstream/handle/10665/43974/978924 1547598 eng.pdf;jsessionid=CCE385357E0FCB553C927B70 E5D7B3CC? sequence=1. abgerufen May 2018.

[26] Hsieh CL, Hsueh IP, Mao HF. (2000). Validity and responsiveness of the rivermead mobility index in stroke patients. Scand J Rehabil Med. (32 (3): 140-2).

[27] Hsueh, I., Wang, C., Sheu, C., \& Hsieh, C. (2003). Comparison of psychometric properties of three mobility measures for patients with stroke. Stroke (34 (7): 1741-5).

[28] Krasny-Pacini, A., Hiebel, J., Pauly, F., Godon, S., \& Chevignard, M. (2013). Goal Attainment Scaling in rehabilitation: A literature-based update. Annals of Physical and Rehabilitation Medicine, 56 (3), 212-230. https://doi.org/10.1016/j.rehab.2013.02.002.

[29] Vloothuis.JDM, van Wegen, E., Veerbek, J., Konijnenbelt, M. V.-M., \& Kwakkel, G. (2015). Caregiver-mediated exercises for improving outcomes after stroke. Cochrane Library (issue 4, No CD0110058).

[30] Lohse, K., Lang, C., \& Boyd, L. (2014). Is more better? Using meta data to explore doese-response relationships in stroke rehabilitation. Stroke (44 (7): 2053-8).

[31] Veerbeek JM, Wegen E Van, Peppen R Van, et al. (2014). What Is the Evidence for Physical Therapy Poststroke ? A Systematic Review and Meta-Analysis. 2014; 9 (2). doi: 10.1371/journal.pone.0087987. 